\title{
Human induced pluripotent stem cells illuminate pathways and novel treatment targets for age-related macular degeneration
}

\author{
Lindsay A. Farrer ${ }^{1}$, Margaret M. DeAngelis ${ }^{2,3}$ \\ ${ }^{1}$ Departments of Medicine (Biomedical Genetics), Neurology, Ophthalmology, Epidemiology, and Biostatistics, Boston University Schools of \\ Medicine and Public Health, Boston, MA, USA; ${ }^{2}$ Department of Ophthalmology and Visual Sciences, John A. Moran Eye Center, ${ }^{3}$ Department of \\ Pharmacotherapy, College of Pharmacy, University of Utah Salt Lake City, UT, USA \\ Correspondence to: Dr. Lindsay A. Farrer. Boston University School of Medicine, Biomedical Genetics E200, 72 East Concord St, Boston, MA 02118, \\ USA. Email: farrer@bu.edu. \\ Provenance: This is an invited Editorial commissioned by Editor-in-Chief Zhizhuang Joe Zhao (Pathology Graduate Program, University of \\ Oklahoma Health Sciences Center, Oklahoma City, USA). \\ Comment on: Saini JS, Corneo B, Miller JD, et al. Nicotinamide ameliorates disease phenotypes in a human iPSC Model of age-related macular \\ degeneration. Cell Stem Cell 2017;20:635-47.e7.
}

Received: 18 October 2017; Accepted: 27 October 2017; Published: 17 November 2017.

doi: $10.21037 /$ sci.2017.10.07

View this article at: http://dx.doi.org/10.21037/sci.2017.10.07

Age-related macular degeneration (AMD) is the most common cause of legal blindness in the United States and is the leading cause of visual impairment in the aging population, especially in those over 55 years of age. By 2020, an anticipated 196 million individuals will be affected with AMD (1). Geographic atrophy is characterized by a slow progressive degeneration of the retinal pigment epithelium (RPE), resulting in the gradual loss of photoreceptors. The wet, or neovascular, form is characterized by the growth of abnormal new blood vessels from beneath the retina that can cause severe and rapid vision loss due to hemorrhage and exudation. Most current treatments are directed against neovascular AMD and are focused against stimulators of angiogenesis (such as vascular endothelial growth factor). These treatments are limited in their applicability, require invasive intravitreal injections, which are burdensome for both patient and physician, and are not capable of preventing or reversing vision loss over the long term. Currently, there are no effective treatments for atrophic AMD.

Significant work in AMD genetics has established CFH and ARMS2/HTRA1 as having large influences on AMD risk in populations of various ethnicities (2-8). Although more than 40 additional AMD-associated risk variants at other loci have been found through case-control association studies with candidate genes (9-12), genomewide association studies of large cohorts $(13,14)$, systems biology $(15,16)$, and studies of pathways shared by multiple disorders (17,18), CFH and ARMS2/HTRA1 genotypes, as well as advanced age, account for most of the known attributable risk for the disorder. Robust AMD associations with common and/or rare variants in $C F H$ and other complement pathway genes including C2/CFB (19), C3 $(9,10), C 9(20), C F I(13,21)$, and VTN (14) suggested that complement inhibition might be a good therapeutic option. However, to date, results of clinical trials using complement inhibitors have not been promising. For example, eculizumab did not improve vision very effectively (22) and lampalizumab had a limited effect in reducing AMD progression $(23,24)$. Recent results reported for the Spectri phase III clinical trial for patients treated with lampalizumab, to reduce lesion their size for geographic atrophy proved to be disappointing (https://www.roche. com/media/store/releases/med-cor-2017-09-08b.htm). Clearly, there is a need to develop more appropriate and effective therapies for AMD, possibly considering the genotype(s) at specific risk loci of the individual when designing a clinical trial toward the goal of personalized medicine (25).

Progress in development of novel effective therapies for AMD based on genetic targets and specific variants could be expedited using rodent model systems, but these models have multiple limitations, most notably the fact that rodents 
don't have maculae to be able to measure pathophysiological endpoints. In a recent article, Saini and colleagues report findings from a series of experiments conducted in human RPE cells, a monolayer in the macula that is essential for photoreceptor support, survival and adversely affected by AMD. These cells were differentiated from human-induced pluripotent stem cells (hiPSCs) derived from eye tissue obtained from patients with documented AMD including two individuals homozygous for AMD risk alleles at the ARMS2/HTRA1 locus (ARMS2/HTRA1+) and from donors without evidence of AMD. Although expression of nine AMD and drusen associated transcripts was not significantly different in the hiPSCs from AMD and healthy control subjects, the authors observed significantly increased levels of transcripts from a set of complement and inflammatory proteins (including $C 3, C F I$, and $C F H$ ) in hiPSCs from AMD subjects. These differences were greatest in comparisons of controls with AMD ARMS2/HTRA1+ subjects.

These experiments alone are noteworthy for at least two reasons. First, although several years have passed since retinal and RPE cells were first derived from human and mouse iPSCs $(26,27)$ and successful submacular transplantation of RPE cells derived in this manner has been performed recently in two patients with neovascular AMD (28), use of transplanted of autologous iPSC-derived RPE cells as a treatment for AMD has many challenges and ultimately may not be effective $(28,29)$. Second, Saini and co-workers provided evidence for the first time that the ARMS2/HTRA1 AMD risk genotype impacts expression of AMD-related proteins, particularly those in the complement system, and thereby provides some clues about how the role of this pathway may function in the disease. However, selection of risk variants from both $A R M S 2$ and HTRA1 as the basis for the ARMS2/HTRA1+ genotype does not allow for the determination of which of these genes actually effects expression of the AMD-related proteins. Nonetheless, this is a solid starting point for future studies with a larger sample size.

The most remarkable aspect of this study was the use of the hiPSC lines to test the therapeutic potential of nicotinamide (NAM), a vitamin B3 derivative with antiinflammatory properties (30) and discover its connections to AMD pathways. Selection of NAM as a therapeutic agent was reasonable in light of evidence showing that elevated serum levels of factors in the vitamin B pathway (vitamin B12, homocysteine, and folate) reduce susceptibility to early and advanced AMD (31). In the current study, it was observed that expression of two AMD- and drusen- associated proteins, clusterin and vascular endothelial growth factor A, was inhibited in hiPSC-RPE cultured cells treated with NAM regardless of the donor group, and this effect was most pronounced in ARMS2/HTRA1+ cells. Secretion of the drusen- and Alzheimer diseaserelated protein $A \beta_{42}$ was inhibited in the NAM-treated AMD but not control hiPSC lines. Next, Saina and coworkers performed bioinformatics analysis to examine the global effects of NAM on the RPE transcriptome. Pathway enrichment analysis suggested that NAM primarily affects the PI3K-Akt signaling and pathway followed by six other pathways including complement and coagulation cascades. Perhaps not surprisingly (and proof of principle), the most significant disease associated gene ontology (GO) terms were macular degeneration and conerod dystrophy. In-depth analysis of genes showing at least nominally significant changes revealed that NAM increased expression of ribosomal synthesis genes in the nucleus and mitochondria, DNA/RNA polymerase, histones, and the gene encoding the sirtuin 1 (SIRT1) protein. Decreased expression of these genes has been shown to be associated with aging $(32,33)$. Further experiments in the hiPSC-RPE lines showed that NAM can effectively decrease inflammatory cytokine production and repress the complement pathway, including $\mathrm{C} 3$.

These experiments underscore the importance of targeting more than one disease pathway or mechanism at a time, while taking into consideration genotype risk. This may be a way forward to obtain more effective AMD therapies as many biochemical pathways overlap and have more than one function.

\section{Acknowledgements}

None.

\section{Footnote}

Conflicts of Interest: The authors have no conflicts of interest to declare.

\section{References}

1. Wong WL, Su X, Li X, et al. Global prevalence of age-related macular degeneration and disease burden projection for 2020 and 2040: a systematic review and meta-analysis. Lancet Glob Health 2014;2:e106-16.

2. Klein RJ, Zeiss C, Chew EY, et al. Complement factor 
$\mathrm{H}$ polymorphism in age-related macular degeneration. Science 2005;308:385-9.

3. Haines JL, Hauser MA, Schmidt S, et al. Complement factor $\mathrm{H}$ variant increases the risk of age-related macular degeneration. Science 2005;308:419-21.

4. Edwards AO, Ritter R 3rd, Abel KJ, et al. Complement factor $\mathrm{H}$ polymorphism and age-related macular degeneration. Science 2005;308:421-4.

5. Jakobsdottir J, Conley YP, Weeks DE, et al. Susceptibility genes for age-related maculopathy on chromosome 10q26. Am J Hum Genet 2005;77:389-407.

6. Rivera A, Fisher SA, Fritsche LG, et al. Hypothetical LOC387715 is a second major susceptibility gene for agerelated macular degeneration, contributing independently of complement factor $\mathrm{H}$ to disease risk. Hum Mol Genet 2005;14:3227-36.

7. Dewan A, Liu M, Hartman S, et al. HTRA1 promoter polymorphism in wet age-related macular degeneration. Science 2006;314:989-92.

8. Yang Z, Camp NJ, Sun H, et al. A variant of the HTRA1 gene increases susceptibility to age-related macular degeneration. Science 2006;314:992-3.

9. Yates JR, Sepp T, Matharu BK, et al. Complement C3 variant and the risk of age-related macular degeneration. N Engl J Med 2007;357:553-61.

10. Maller JB, Fagerness JA, Reynolds RC, et al. Variation in complement factor 3 is associated with risk of age-related macular degeneration. Nat Genet 2007;39:1200-1.

11. Ennis S, Jomary C, Mullins R, et al. Association between the SERPING1 gene and age-related macular degeneration: a two-stage case-control study Lancet 2008;372:1828-34.

12. Jun G, Nicolaou M, Morrison MA, et al. Influence of ROBO1 and RORA on risk of age-related macular degeneration reveals genetically distinct phenotypes in disease pathophysiology. PLoS One 2011;6:e25775.

13. Fritsche LG, Chen W, Schu M, et al. Seven new loci associated with age-related macular degeneration. Nat Genet 2013;45:433-9.

14. Fritsche LG, Igl W, Bailey JN, et al. A large genomewide association study of age-related macular degeneration highlights contributions of rare and common variants. Nat Genet 2016;48:134-43.

15. Silveira AC, Morrison MA, Ji F, et al. Convergence of linkage, gene expression and association data demonstrates the influence of the RAR-related orphan receptor alpha (RORA) gene on neovascular AMD: a systems biology based approach. Vision Res 2010;50:698-715.
16. Morrison MA, Silveira AC, Huynh N, et al. Systems biology-based analysis implicates a novel role for vitamin $\mathrm{D}$ metabolism in the pathogenesis of age-related macular degeneration. Hum Genomics 2011;5:538-68.

17. Logue MW, Schu M, Vardarajan BN, et al. Search for age-related macular degeneration risk variants in Alzheimer disease genes and pathways. Neurobiol Aging 2014;35:1510.e7-18.

18. Grassmann F, Kiel C, Zimmermann ME, et al. Genetic pleiotropy between age-related macular degeneration and 16 complex diseases and traits. Genome Med 2017;9:29.

19. Gold B, Merriam JE, Zernant J, et al. Variation in factor $\mathrm{B}(\mathrm{BF})$ and complement component 2 (C2) genes is associated with age-related macular degeneration. Nat Genet 2006;38:458-62.

20. Seddon JM, Yu Y, Miller EC, et al. Rare variants in CFI, C3 and C9 are associated with high risk of advanced age-related macular degeneration. Nat Genet 2013;45:1366-70.

21. van de Ven JP, Nilsson SC, Tan PL, et al. A functional variant in the $\mathrm{CFI}$ gene confers a high risk of age-related macular degeneration. Nat Genet 2013;45:813-17.

22. Yehoshua Z, de Amorim Garcia Filho CA, Nunes RP, et al. Systemic complement inhibition with eculizumab for geographic atrophy in age-related macular degeneration: the COMPLETE study. Ophthalmology 2014;121:693-701.

23. Rhoades W, Dickson D, Do DV. Potential role of lampalizumab for treatment of geographic atrophy. Clin Ophthalmol 2015;9:1049-56.

24. Volz C, Pauly D. Antibody therapies and their challenges in the treatment of age-related macular degeneration. Eur J Pharm Biopharm 2015;95:158-72.

25. DeAngelis MM, Owen LA, Morrison MA, et al. Genetics of age-related macular degeneration (AMD). Hum Mol Genet 2017;26:R246.

26. Hirami Y, Osakada F, Takahashi K, et al. Generation of retinal cells from mouse and human induced pluripotent stem cells. Neurosci Lett 2009;458:126-31.

27. Kamao H, Mandai M, Okamoto S, et al. Characterization of human induced pluripotent stem cell-derived retinal pigment epithelium cell sheets aiming for clinical application. Stem Cell Reports 2014;2:205-18.

28. Mandai M, Watanabe A, Kurimoto Y, et al. Autologous induced stem-cell-derived retinal cells for macular degeneration. N Engl J Med 2017;376:1038-46.

29. Souied E, Pulido J, Staurenghi G. Autologous induced stem-cell-derived retinal cells for macular degeneration 
(letter). N Engl J Med 2017;377:792.

30. Maiese K, Chong ZZ, Hou J, et al. The vitamin nicotinamide: translating nutrition into clinical care. Molecules 2009; 14:3446-85.

31. Gopinath B, Flood VM, Rochtchina E, et al. Homocysteine, folate, vitamin B-12, and 10-y incidence of age-related macular degeneration. Am J Clin Nutr 2013;98:129-35.

32. López-Otín C, Blasco MA, Partridge L, et al. The hallmarks of aging. Cell 2013;153:1194-217.

33. Pal S, Tyler JK. Epigenetics and aging. Sci Adv 2016;2:e1600584. doi: $10.21037 /$ sci.2017.10.07

Cite this article as: Farrer LA, DeAngelis MM. Human induced pluripotent stem cells illuminate pathways and novel treatment targets for age-related macular degeneration. Stem Cell Investig 2017;4:92. 